Physica 148A (1988) 312-330

North-Holland, Amsterdam

\title{
TIME-ASYMPTOTICS AND THE SELF-ORGANIZATION HYPOTHESIS FOR 2D NAVIER-STOKES EQUATIONS
}

\author{
E. VAN GROESEN \\ Department of Applied Mathematics, University of Twente, P.O. Box 217. \\ 7500 AE Enschede, The Netherlands \\ Received 25 May 1987
}

\begin{abstract}
In this paper we study the long-time behaviour of solutions with a two-dimensional structure of the Navier-Stokes equations on a periodic grid. From a rigorous investigation of the decrease of the energy $E$ and the enstrophy $W$, it follows that the Rayleigh quotient $Q=W / E$ is monotonically decreasing on solutions. This is shown to imply that the spatial structure of any solution tends to a critical point of $Q$, which is the structure of some planar vortex, and that all these structures are unstable except for the fundamental one which has the longest wavclength. The time dependence of the approach towards this seif-organized state is investigated in some detail. In the spectral plane, the mean-squared wave-numbers of both the spectral energy- and enstrophy density are shown to decrease as a function of time. For the enstrophy this implics that the normal cascade does not match the discriminating effect of the viscous dissipation.
\end{abstract}

\section{Introduction}

In this paper we investigate the time-asymptotic behaviour of solutions on a periodic grid of the incompressible Navier-Stokes equations with uniform density $\rho=1$ :

$$
\begin{aligned}
& \boldsymbol{v}_{t}=-(\boldsymbol{v} \cdot \boldsymbol{\nabla}) \boldsymbol{v}+\nabla p+\nu \Delta v, \\
& \boldsymbol{\nabla} \cdot \boldsymbol{v}=0
\end{aligned}
$$

Here $v$ is the fluid velocity, $p$ the pressure and $\nu>0$ the kinematic viscosity.

The paper by Foias and Saut ${ }^{3}$ ) deals with this asymptotic behaviour for both two- and three-dimensional flows. In this paper we shall show that for $2 \mathrm{D}$ flows some more information can be obtaincd, especially concerning the stability of the spectral manifolds introduced in ref. 3 , and the rate of convergence of the spatial structure to the fundamental manifold.

Besides this, a main point of this paper is to show that the mathematical methods to obtain the results can be considered to be inspired by the so-called 
"self-organization hypothesis" which is known in the physical literature and that, on the other hand, the mathematical treatment illuminates and specifies some of the physical ideas that underly the hypothesis.

Briefly, the self-organization hypothesis can be described as follows (see e.g. Hasegawa ${ }^{4}$ ) and the references thercin; this paper raised many intriguing questions, and increased the motivation for this research).

Assume that a dissipative system, when dissipation is neglected, posseses at least two constants of the motion, say $A$ and $B$. Critical points of $A$ on level sets of $B$ then form a set that is invariant for the conservative system. These critical points satisfy for some Lagrange multiplier $\lambda$ the equation

$$
\frac{\delta A}{\delta u}=\lambda \frac{\delta B}{\delta u} .
$$

We will call these solutions steady states since in many cases they are timeindependent solutions of the conservative system or correspond to travellingwave-like solutions. In the presence of dissipation, the functionals $A$ and $B$ will not be conserved in general. Now assume that one of these functionals, say $A$, dissipates faster than the other one, $B$ (selective dissipation of the functionals). The self-organization hypothesis then, loosely, states that the asymptotic behaviour of the solutions of the dissipative system is related to the solutions of the constrained minimization problem of minimizing $A$ on level sets of $B$.

We will not go into a more precise statement of the hypothesis at this place. We merely note that the argumentation for the selective dissipation property as found in the literature is quite complicated and not completely convincing, and that the statements about the asymptotics are not very precise (compared to the results of Foias and Saut ${ }^{3}$ ) for instance). The main reason seems to be that most of the analysis takes place in the spectral plane, where the flow of the spectral densities of the functionals are investigated. Upon averaging over ensembles, non-linear mode interactions (leading to normal or inverse cascade) and dissipative effects are considered to conclude the selective dissipation of the densities in specific cases.

In this paper we will deal with the deterministic equations directly and derive from them the selective dissipation of the functionals themselves. From the limiting behaviour of these functionals we are able to obtain precise results on the asymptotics of solutions. Notwithstanding the deterministic approach, some results have a specific meaning for the behaviour in the spectral plane. For the $\mathrm{N}-\mathrm{S}$ equations (1.1), the functionals that will play the rôle of $A$ and $B$ are the enstrophy and energy, respectively. See Rhines ${ }^{10}$ ), Bretherton and Haidvogel $^{1}$ ), Yoishida et al ${ }^{12}$ ) and references therein for the idea of self-organization in this case. For recent results on linearization and normal form of the $\mathrm{N}-\mathrm{S}$ equations see ref. 2. 
After some preliminaries in section 2 we discuss the steady states for the Euler equations $(\nu=0)$ in section 3 and the corresponding dissipating vortices for the $\mathrm{N}-\mathrm{S}$ equations in section 4 . In the next section we describe how the evolution of the system can be envisaged in the $E-W$ plane, and section 6 shows that the quotient $W / E$ is monotonically decreasing in time and that the possible limiting values define invariant sets, as in ref. 3 , the stability of which can be investigated. The results of the asymptotic behaviour are described in section 7 and are proved in section 9. Section 8 deals with the spectral interpretation of some of the results.

\section{Preliminaries}

It is customary to eliminate the pressure from the momentum equation (1.1) by taking the curl of this equation. Restricting ourselves to planar flows, with the vorticity $\boldsymbol{\omega}=\operatorname{curl} \boldsymbol{v}=(0,0, \omega)$ the equations become

$$
\begin{aligned}
& \omega_{t}=-\boldsymbol{v} \cdot \nabla \omega+\nu \Delta \omega, \\
& \nabla \cdot \boldsymbol{v}=0 .
\end{aligned}
$$

In order to find a spatial periodic structure, one might choose periodic boundary conditions for $\boldsymbol{v}$ on an elementary cell in the $x-y$ plane. However, then 1D flow structures will appear along with the $2 \mathrm{D}$ structures. In order to avoid 1D structures, choose as domain of definition the quarter cell $R=$ $(0, \pi / a) \times(0, \pi / b)$ and boundary conditions such that an appropriate continuation of a solution on $R$ will produce a smooth periodic grid on $\mathbb{R}^{2}$ with $R$ as a quarter cell. Appropriate boundary conditions turn out to be

$$
\begin{array}{ll}
\boldsymbol{v} \cdot \boldsymbol{n}=0 & \text { on } \partial R, \\
\omega=0 \quad \text { on } \partial R,
\end{array}
$$

as can be easily verified. Note that the flow is completely described by the vorticity $\omega$ when $\boldsymbol{v}$ is determined subject to (2.2). Therefore we will consider all the functionals as they appear below as functionals of $\omega$. Another description is possible with Stokes stream function $\psi$ defined by $\psi=0$ on $\partial R$ and $\boldsymbol{v}=\boldsymbol{\nabla}^{\perp} \psi=\left(\psi_{y},-\psi_{x}, 0\right)$, for which $\omega=-\Delta \psi$.

The energy $E$ and the enstrophy $W$ are defined by

$$
E(\omega)=\frac{1}{2} \int \boldsymbol{v}^{2}
$$


and

$$
W(\omega)=\frac{1}{2} \int \omega^{2}
$$

respectively, and these are constants of the motion for the Euler equations, i.e. for (2.1), or (1.1), with $\nu=0$.

\section{Steady states}

According to the self-organization hypothesis, let us first look at the steady states determined by $E$ and $W$, i.e. at the solutions of the constrained variational principle for critical points of $W$ on level sets of $E$. This variational problem will be denoted by

$$
\operatorname{crit}\{W(\omega) \mid E(\omega)=\gamma ; \omega=0 \text { on } \partial R\},
$$

where $\gamma>0$ is the prescribed value of the energy.

Since both $W$ and $E$ are quadratic functionals, these solutions are simply a multiplicative sealing factor $\sqrt{\gamma}$ times the solution of the normalized problem with $\gamma=1$ in (3.1), and are also the same, up to scaling, as the critical points of the Rayleigh quotient:

$$
\operatorname{crit}\{Q(\omega) \mid \omega=0 \text { on } \partial R, \omega \neq 0\} \text {, }
$$

where

$$
Q(\omega)=W(\omega) / E(\omega)
$$

These critical points are precisely the solutions of an eigenvalue problem which, in terms of $\omega$, is simply

$$
\begin{array}{ll}
-\Delta \omega=\lambda \omega & \text { on } R, \\
\omega=0 & \text { on } \partial R .
\end{array}
$$

The eigenvalues and eigenfunctions can be written down explicitly: for $\boldsymbol{k}=$ $\left(\boldsymbol{k}_{1}, \boldsymbol{k}_{2}\right) \in \mathbb{N} \times \mathbb{N}$

$$
\lambda_{k}=a k_{1}^{2}+b k_{2}^{2} \text { and } \hat{\Omega}_{k}=\frac{2 \sqrt{a b}}{\pi} \sin (a k x) \sin (b k y),
$$


where the hat ${ }^{\wedge}$ indicates that $\hat{\Omega}_{k}$ has been normalized for later convenience such that $\left\|\hat{\Omega}_{k}\right\|=1$, where \|\| is the usual $\mathrm{L}$,-norm; thus

$$
E\left(\hat{\Omega}_{k}\right)=\frac{1}{2} \frac{1}{\lambda_{k}} \quad \text { and } \quad W\left(\hat{\Omega}_{k}\right)=\frac{1}{2} .
$$

As is well known, the set $\hat{\Omega}_{k}$ forms a complete, orthogonal set of functions. Moreover, denote the increasing set of eigenvalues by $\mu_{k}, k \in \mathbb{N}$ :

$$
0<\mu_{1}=\lambda_{(1,1)}<\mu_{2}<\mu_{3}<\cdots
$$

and the eigenspace corresponding to $\mu_{k}$ by $\mathrm{E}_{k}$. Then the smallest eigenvalue $\mu_{1}$ is simple, i.e. $E_{1}$ is 1 -dimensional and spanned by $\hat{\Omega}_{1}:=\hat{\Omega}_{(1.1)}$, and $\mu_{1}$ admits the extremizing characterization

$$
\mu_{1}=\min \{Q(\omega) \mid \omega=0 \text { on } \partial R\}=Q\left(\hat{\Omega}_{1}\right)
$$

For future reference we denote the velocity fields corresponding to $\hat{\Omega}_{k}$ by $V_{k}$; explicitly, $V_{k}$ is given by

$$
\lambda_{k} V_{k}=\nabla^{\perp} \hat{\Omega}_{k}
$$

Remark 3.1. Define a functional $\Lambda(\omega)$ by

$$
\Lambda(\omega)=\frac{D(\omega)}{W(\omega)}=\frac{\int(\nabla \omega)^{2}}{\int \omega^{2}},
$$

where

$$
D(\omega)=\frac{1}{2} \int(\nabla \omega)^{2}
$$

Then the critical points of $\Lambda$ give rise to the same eigenvalue problem (3.4). Consequently, the functionals $\Lambda$ and $Q$ have the same critical points $\hat{\Omega}_{k}$ and $\Lambda\left(\hat{\Omega}_{k}\right)=Q\left(\hat{\Omega}_{k}\right)$. This functional $\Lambda$ will admit a definite physical interpretation in the following.

Since both $E$ and $W$ are constants of the motion for the Euler equation, the following proposition follows from general results (see $\mathrm{Lax}^{8}$ ), a generalization of an idea due to Kelvin ${ }^{5}$ ) or from a simple direct verification. 
Proposition 3.2. Any $\Omega \in \mathrm{E}_{k}, k \in \mathbb{N}$ is a time-independent solution of the Euler equations.

This completes the analysis of the steady states determined by the functionals $E$ and $W$.

Remark 3.3. Apart from $W$ defined by (2.5), the so-called generalized enstrophy

$$
\Phi(\omega):=\int f(\omega)
$$

is for arbitrary function $f$ also a constant of the motion for the Euler equations. The constrained variational problem for $\Phi$ and $E$ gives rise to many more steady states, which satisfy a non-linear eigenvalue problem. These states are completely different from the states obtained above if $f$ is not quadratic. The asymptotic analysis that follows will clarify and justify the particular choice of the enstrophy $W$.

\section{Planar Taylor vortices}

It is a peculiar fact that the equation determining the steady states, i.e. (3.4), and the specific form of the viscosity term in (2.1) are such that they admit for exact solutions of the complete equation (2.1).

Proposition 4.1. For any $\Omega \in \mathrm{E}_{k}$, the function

$$
\Omega \exp \left(-\nu \mu_{k} t\right)
$$

is an exact solution of the Navier-Stokes equations (2.1).

After Taylor ${ }^{11}$ ), we will call these solutions planar Taylor vortices.

Remark 4.2. Note that, as a consequence of the unique solvability of the initial value problem, the vortices (4.1) are the only motions that are possible in $\mathrm{E}_{k}$. More generally, if $\omega(t)$ is a solution for which $\omega\left(t_{0}\right) \in \mathrm{E}_{k}$ for some $t_{0}$ and some $k \in \mathbb{N}$, then $\omega(t)=\omega\left(t_{0}\right) \exp \left(-\nu \mu_{k}\left(t-t_{0}\right)\right)$ for all $t$, and hence $\omega$ is a planar Taylor vortex. 


\section{Motion in the $E-W$ diagram}

The evolution of a solution can be partly envisaged in a simple way by looking at its successive values of $E$ and $W$. As will become clear in the following, this restricted picture of the motion is helpful to illuminate the analysis and to interpret the self-organization hypothesis.

In the $E-W$ plane the evolution of the vorticity $\omega=\omega(t)$ as described by (2.1) defines a continuous curve

$$
t \rightarrow(E(\omega(t)), W(\omega(t)))
$$

The time-derivatives of $E$ and $W$ at a solution are known to be given by

$$
\dot{E}(\omega)=-2 \nu W(\omega)
$$

and

$$
\dot{W}(\omega)=-2 \nu D(\omega)
$$

where $D(\omega)$ is the functional introduced in $(3.10)$. Consequently, the functional $\Lambda(\omega)$ introduced in (3.9) satisfies

$$
\Lambda(\omega)=\dot{W}(\omega) / \dot{E}(\omega)
$$

and therefore admits the interpretation of being the dissipation rate quotient, measuring the relative rate of dissipation of the functionals $W$ and $E$ due to viscosity.

In the $E-W$ diagram, the functionals $Q$ and $A$ have a clear meaning: at a point of the curve (5.1), $Q$ determines the angle of the point with the positive $E$-axis, while $A$ is the tangent to the curve at this point.

Note that since both $A$ and $Q$ are homogenous of degree 0 , their value at some $\omega$ is only determined by the spatial structure, and not by the amplitude of $\omega$. That is to say, if we write $\hat{\omega}$ for the spatial structure of $\omega$, defined by

$$
\hat{\omega}=\frac{\omega}{\|\omega\|},
$$

where \|\| is the usual $L_{2}$-norm, then

$$
Q(\omega)=Q(\hat{\omega}) \text { and } \Lambda(\omega)=\Lambda(\hat{\omega}) \text { for all } \omega
$$


The eigenvalues $\mu_{k}$ determine straight lines in the E-W diagram going through the origin and with $Q=\mu_{k}$. Each time-independent solution $\Omega \in \mathrm{E}_{k}$ of the Euler equations corresponds to a fixed point on this line. Under the influence of viscosity, the planar Taylor vortex (4.1) corresponds to a motion along this line directed towards the origin as a consequence of the facts that $Q\left(\Omega \exp \left(-\nu \mu_{k} t\right)\right)=Q(\Omega)=\mu_{k}$ and $\dot{E}(\omega)<0$.

Note that from the extremizing characterization (3.8) for $\mu_{1}$ it follows that no points in the $E-W$ diagram below the $Q=\mu_{1}$ line correspond to physically realistic states $\omega$, while the only points on the line $Q=\mu_{1}$ are planar Taylor vortices with $\mu_{1}$.

From (5.2) and (5.3) it readily follows that both $E$ and $W$ decrease exponentially fast as $t \rightarrow \infty$. In fact, since $D(\omega) \geqslant \mu_{1} W(\omega)$ for all $\omega$, it follows from (5.3) that

$$
\dot{W}=-2 \nu D \leqslant-2 \nu \mu_{1} W,
$$

and thus

$$
W(\omega(t)) \leqslant W(\omega(0)) \exp \left(-2 \nu \mu_{1} t\right)
$$

In the same way it follows with $W(\omega) \geqslant \mu_{1} E(\omega)$ that

$$
E(\omega(t)) \leqslant E(\omega(0)) \exp \left(-2 \nu \mu_{1} t\right) .
$$

\section{Viscous evolution of $Q$ and invariant sets}

We will now consider the behaviour of $Q$ along a solution. Because of the restriction to two-dimensional flow the change in $Q$ is only due to viscosity and we shall see that $Q$, in fact, decreases monotonically. The monotonicity will also allow us to determine the stability of invariant sets that are characterized by the limiting value of $Q$.

With (5.2) and (5.3) the expression for $\dot{Q}$ can be written in a nice way by using the functional $\Lambda$ introduced in (3.9):

$$
\dot{Q}=-2 \nu Q[\Lambda-Q] \text {. }
$$

Since $Q$ is positive, the sign of $\dot{Q}$ is determined by $A-Q$.

Lemma 6.1. For each $\omega$ with $\omega=0$ on $\partial R$ the expression $A-Q$ is signdefinite, 


$$
\Lambda(\omega)-Q(\omega) \geqslant 0,
$$

and, moreover,

$$
A(\omega)-Q(\omega)=0 \text { iff } \omega \in \mathrm{E}_{k} \text { for some } k \in \mathbb{N} .
$$

Proof. Using Stokes stream function $\psi$, note that $\int \boldsymbol{v}^{2}=\int\left(\nabla^{\perp} \psi\right)^{2}=\int(\nabla \psi)^{2}$ and $\int \omega^{2}=\int(-\Delta \psi) \omega=\int \nabla \psi \cdot \nabla \omega$. Then the first statement is an easy consequence of the Canchy-Schwartz inequality:

$$
\left(\int \psi^{2}\right)^{2}=\left(\int \boldsymbol{\nabla} \psi \cdot \nabla \omega\right)^{2} \leqslant \int(\nabla \psi)^{2} \cdot \int(\nabla \omega)^{2}=\int \boldsymbol{v}^{2} \cdot \int(\nabla \omega)^{2},
$$

and hence $W^{2} \leqslant E \cdot D$, i.e. $A-Q \geqslant 0$. Moreover, equality holds iff $\nabla \psi$ and $\nabla \omega$ are colinear: $\nabla \omega=\sigma \nabla \psi$ for some $\sigma \in \mathbb{R}$. With $\omega=-\Delta \psi$ this implies that $\omega$ is an eigenfunction of (3.4) and hence $\omega \in \mathrm{E}_{k}$ for some $k \in \mathbb{N}$.

Since $Q$ satisfies $Q \geqslant \mu_{1}$, it follows from (6.1) and the lemma that $Q$ decreases monotonically for any solution that is not planar Taylor vortex. Being bounded from below, $Q(\omega(t))$ necessarily has a limit as $t \rightarrow \infty$. This limit, which only depends on the initial condition $\omega(0)$, shall be denoted by $q(\omega(0))$. Furthermore, since $Q$ decreases monotonically, $\dot{Q} \rightarrow 0$ as $t \rightarrow \infty$ and hence $\Lambda(\omega(t))-Q(\omega(t)) \rightarrow 0$ as $t \rightarrow \infty$.

Corollary 6.2. For the solution $\omega(t)$ with initial condition $\omega(0), \dot{Q}(\omega(t))$ is non-positive and the limit

$$
q(\omega(0)):=\lim _{t \rightarrow r} Q(\omega(t))
$$

exists and satisfies $\mu_{1} \leqslant q(\omega(0)) \leqslant Q(\omega(0))$, with $q(\omega(0))=Q(\omega(0))$ iff $\omega(0) \in$ $\mathrm{E}_{k}$ for some $k \in \mathbb{N}$, i.e. iff $\omega(t)$ is some planar Taylor vortex. Moreover, it also holds that

$$
q(\omega(0))=\lim _{t \rightarrow \infty} \Lambda(\omega(t)) .
$$

The next result states that the possible limiting values $q(\omega(0))$ are quantizized.

Proposition 6.3. (Ref. 3.) for each initial condition $\omega(0)$ there is some $k \in \mathbb{N}$ such that $q(\omega(0))=\mu_{k}$, where $\mu_{k}$ is one of the eigenvalues (3.7).

Proof. The proof is somewhat simpler than that in ref. 3 since we can use the additional information (6.5). We shall show that for any solution $\omega(t)$ and any 
time sequence $t_{n} \rightarrow \infty$ the corresponding sequence of normalized spatial structures $\hat{\omega}_{n}:=\hat{\omega}\left(t_{n}\right)$ has a subsequence that converges to some limit $\hat{\omega}$ for which $\Lambda(\hat{\omega})=Q(\hat{\omega})=q(\omega(0))$. From $\Lambda(\hat{\omega})=Q(\hat{\omega})$ it then follows with (6.3) that $\hat{\omega} \in \mathrm{E}_{k}$ for some $k \in \mathbb{N}$, and then $q(\omega(0))=\mu_{k}$. (Note that it requires some more elaboration to show that $\hat{\omega}=\lim _{t \rightarrow \infty} \hat{\omega}(t)$, i.e. that $\hat{\omega}$ is the unique limit, independent of the time sequence. This will be the statement in the next section and proved in section 9.)

The quadratic functionals $E, W$ and $D$ define three (inner product) norms on the function space, the closure of which thus provides three Hilbert spaces which are compactly embedded:

$$
\mathrm{H}_{D} \hookrightarrow \mathrm{H}_{W} \hookrightarrow \mathrm{H}_{E}
$$

( $\mathrm{H}_{W}$ is the usual $\mathrm{L}_{2}$-space, $\mathrm{H}_{D}$ is a Sobolev space of order 1 and $\mathrm{H}_{E}$ is the dual of $\mathrm{H}_{D}$, the Sobolev space of negative order 1.) By the normalization of $\hat{\omega}_{n}$ it follows that $W\left(\hat{\omega}_{n}\right)=\frac{1}{2}$, and thus with (6.5) that $D\left(\hat{\omega}_{n}\right)=\frac{1}{2} \Lambda\left(\hat{\omega}_{n}\right)$ is bounded. Standard Hilbert space theory then implies that $\hat{\omega}_{n}$ has a subsequence that converges weakly in $\mathrm{H}_{D}$ to some $\hat{\omega} \in \mathrm{H}_{D}$. By compactness, this subsequence converges strongly in $\mathrm{H}_{W}$ and $\mathrm{H}_{E}$. Consequently, $\|\hat{\omega}\|=\lim \left\|\hat{\omega}_{n}\right\|=1$, i.e. $\hat{\omega}$ is a normalized spatial structure, and $Q(\hat{\omega})=\lim Q\left(\hat{\omega}_{n}\right)=q(\omega(0))$. The weak convergence in $\mathrm{H}_{D}$ implies $\Lambda(\hat{\omega})=2 D(\bar{\omega}) \leqslant \lim \inf 2 D\left(\hat{\omega}_{n}\right)=\lim \inf A\left(\hat{\omega}_{n}\right)=$ $q(\omega(0))$. With (6.2) it then follows $q(\omega(0))=Q(\hat{\omega}) \leqslant \Lambda(\hat{\omega}) \leqslant q(\omega(0))$, and thus $Q(\hat{\omega})=\Lambda(\hat{\omega})$, which is what we set out to prove.

The foregoing proposition makes it possible to define sets that are invariant for the flow. Let, for $k \in \mathbb{N}$,

$$
I_{k}:=\left\{\omega \mid q(\omega)=\mu_{k}\right\}
$$

Then, clearly, $I_{k}$ is invariant, contains $\mathrm{E}_{k}$ and the intersection with any $I_{j}$, $j \neq k$, is empty while the union of all $I_{k}$ 's is the whole function space.

These sets $I_{k}$ are closely related to the invariant manifolds introduced in ref. 3. It seems difficult to describe the sets $I_{k}$ in a more explicit way. The next result specifies a rather large subset of $I_{1}$ and indicates that from some practical point of view, the set $I_{1}$ is the only "stable" one.

Proposition 6.4. The invariant set $I_{1}$ contains the following subsets:

$$
\left\{\omega \mid Q(\omega)<\mu_{2}\right\} \quad \text { and } \quad\left\{\omega \mid Q(\omega)=\mu_{2}, \omega \notin \mathrm{E}_{2}\right\} .
$$

Moreover, each of the sets $I_{k}$ with $k \neq 1$ is asymptotically unstable in the following sense: if $\omega(t) \in I_{k}, k \neq 1$, then, given any arbitrary small perturbation 
$\xi \in \mathrm{E}_{1} \cup \mathrm{E}_{2} \cup \ldots \cup \mathrm{E}_{k-1}$, there exists $T>0$ (sufficiently large) such that the solution with initial condition $\omega(T)+\xi$ belongs to $I_{j}$ for some $j<k$.

Proof. That the sets (6.7) are contained in $I_{1}$ is immediate from corollary 6.2. To show the instability result we use the fact that $E(\omega(t))$ and $W(\omega(t))$ tend to zero, cf. (5.5), (5.6), and that for $\xi \in \mathrm{E}_{1} \cup \mathrm{E}_{2} \cup \ldots \cup \mathrm{E}_{k-1}$ it holds that $W(\xi) \leqslant \mu_{k-1} E(\xi)$, i.e. $Q(\xi) \leqslant \mu_{k-1}<\mu_{k}$. Since

$$
Q(\omega+\xi)=\frac{W(\omega+\xi)}{E(\omega+\xi)}=\frac{W(\xi)+o(\|\omega\|)}{E(\xi)+o(\|\omega\|)}
$$

for $\|\omega\| \rightarrow 0$, it follows that, provided $T$ is sufficiently large, $Q(\omega(T)+\xi)<$ $\mu_{k}$. The monotonic decrease of $Q$ then implics $q(\omega(T)+\xi)<\mu_{k}$, and the conclusion follows.

Remark 6.5. It is instructive to visualize the results of this section in the $E-W$ diagram and to see that each curve (5.1) approaches some $Q=\mu_{k}$ line from above and is tangent to it at the origin. By a suitable perturbation such a limiting line (with $k \neq 1$ ) can be crossed after which (in the absence of further perturbations) the decrease of $Q$ forces an asymptotic approach to some lower lying line $Q=\mu_{j}$ with $j<k$.

\section{Asymptotic spatial structure}

Having found that the quantity $Q(\omega(t))$ has some eigenvalue $\mu_{k}$ as limiting asymptotic value, we will now state that then $\omega(t)$ behaves asymptotically as a planar vortex in $\mathrm{E}_{k}$. Moreover, for the physically most interesting case, i.e. $k=1$ which is the stable case according to the previous results, we will present an estimate for the rate of convergence to the fundamental vortex solution.

In the following we will denote the orthogonal complement (in the $\mathrm{L}_{2}$ sense) of $\mathrm{E}_{k}$ by $\mathrm{E}_{k}^{\perp}$, i.e. $\mathrm{E}_{k}^{\perp}=\left\{\xi \mid \int \xi \cdot \Omega=0\right.$ for all $\left.\Omega \in \mathrm{E}_{k}\right\}$.

Proposition 7.1. Let $\omega(t)$ be a solution which belongs to $I_{k}$ for some $k$, and write

$$
\omega(t)=\Omega(t)+\xi(t), \text { with } \Omega(t) \in \mathrm{E}_{k} \text { and } \xi(t) \in \mathrm{E}_{k}^{\perp} .
$$

Then we have

(i) $\|\xi(t)\| /\|\Omega(t)\| \rightarrow 0$ as $t \rightarrow \infty$; 
(ii) there is some $\hat{\Omega} \in \mathrm{E}_{k}$ such that $\hat{\Omega}(t) \mathrm{e}^{\nu \mu_{k} t} \rightarrow \hat{\Omega}$ as $t \rightarrow \infty$.

Moreover, in case $k=1$, the result (i) can be specified as follows:

$$
\text { (i*) } \frac{\|\xi(t)\|}{\|\Omega(t)\|} \leqslant c \exp (-\nu \sigma t)
$$

for any number $\sigma$ satisfying $\sigma \leqslant\left(\mu_{2}-\mu_{1}\right)^{2} / \mu_{2}$ and some constant $c>0$.

We will present a proof of these results in section 9. In order to interpret this result in the context of the self-organization hypothesis we first make the following immediate consequence explicit.

Corollary 7.2. Each solution $\omega(t)$ has an asymptotic spatial structure:

$$
\lim _{t \rightarrow \infty} \hat{\omega}(t)=\lim _{t \rightarrow \infty} \frac{\omega(t)}{\|\omega(t)\|}=\hat{\Omega}
$$

exists and $\hat{\Omega} \in \mathrm{E}_{k}$ for $\omega \in I_{k}$.

This corollary specifies the statement in the self-organization hypothesis as described in the introduction. More precisely, 7.2 states that the asymptotic spatial structure is a critical point of the enstrophy on a level set of the energy. This critical point is in fact a minimizer (as in the usual statement in the formulation of the hypothesis) in case $k=1$ and, in view of the instability, in the other cases in the presence of perturbations with the longest wavelength, i.e. for perturbations from $E_{1}$.

Remark 7.3. The asymptotic results above do not explain the appearance of small-scaled vortices as has been observed in recent computer simulations by McWilliams ${ }^{9}$ ).

\section{Averaged spectral energy and enstrophy flows}

In this section we will show that the results of the foregoing sections can be translated into statements about the spectral energy and enstrophy density. Since the results were derived directly from the deterministic equations and were based on the evolution of functionals instead of densities, we will find conclusions for averaged spectral quantities only, averaging over all wavenumbers. Consequently, results about normal or inverse cascade of spectral densities cannot be expected. Nevertheless, the conclusions about the averaged 
densities show the averaged effect of both the nonlinearity of the system and of the dissipation due to viscosity.

Since the eigenfunctions $\Omega_{k}, k \in \mathbb{N}^{2}$ form a complete set of functions, any $\omega$ can be written like

$$
\omega=\sum_{k} \alpha_{k} \Omega_{k}, \quad \alpha_{k} \in \mathbb{R} .
$$

The corresponding velocity is then given by

$$
v=\sum_{k} \alpha_{k} V_{k}
$$

For convenience we assume in this section that $V_{k}$ is normalized such that $\left\|V_{k}\right\|=1$, and hence $\left\|\Omega_{k}\right\|^{2}=\lambda_{k}$. Using the orthogonality of the sets $\left\{\Omega_{k}\right\}_{k}$ and $\left\{V_{k}\right\}_{k}$ and this normalization, the functionals $Q$ and $\Lambda$ can then be expressed in terms of the Fourier coefficients $\alpha_{k}$ like

$$
Q(\omega)=\frac{\sum \lambda_{k} \alpha_{k}^{2}}{\sum \alpha_{k}^{2}} \text { and } A(\omega)=\frac{\sum \lambda_{k}^{2} \alpha_{k}^{2}}{\sum \lambda_{k} \alpha_{k}^{2}} .
$$

In the spectral plane $\lambda_{k}=a^{2} k_{1}^{2}+b^{2} k_{2}^{2}=\left|\left(a k_{1}, b k_{2}\right)\right|^{2}$ is the square of the distance from the origin to the grid point $\left(a k_{1}, b k_{2}\right)$.

Introducing radial (omnidirectional) energy and enstrophy densities by

$$
e_{i}:=\sum_{\substack{k \\ \lambda_{k}=\mu_{i}}} \alpha_{k}^{2} \quad \text { and } \quad w_{i}:=\sum_{\substack{k \\ \lambda_{k}=\mu_{i}}} \lambda_{k} \alpha_{k}^{2},
$$

the functionals $Q$ and $A$ can be written as

$$
Q(\omega)=\frac{\sum \mu_{i} e_{i}}{\sum e_{i}} \text { and } \Lambda(\omega)=\frac{\sum \mu_{i} w_{i}}{\sum w_{i}},
$$

respectively.

This shows that $Q(\omega)$ and $\Lambda(\omega)$ can be interpreted as the mean-squared wavenumber with weight function the spectral energy- and enstrophy density, respectively. As such, $Q(\omega)$ and $\Lambda(\omega)$ are good measures of the relative contribution to the total energy and enstrophy, respectively, of the different Fourier components that are present in the function $\omega$.

Consequently, the time-behaviour of $Q(\omega(t))$ and $A(\omega(t))$ gives a description of the change of the wavenumbers that predominantly contribute to the total energy and enstrophy at a certain time. 
The results of the foregoing sections will now be used to investigate these averaged spectral energy and enstrophy flows.

To start with, we consider the energy. For planar Taylor vortices, $Q$ is constant, $\mu_{k}$ for some $k \in \mathbb{N}$, and there is no spectral energy flow. For all other solutions, $Q(\omega(t))$ has been shown to decrease monotonically to $\mu_{k}$ for some $k \in \mathbb{N}$. Hence, the mean-squared wavenumber for the energy density decreases monotonically and the possible limiting values are quantizized, viz. belong to the set of eigenvalues $\mu_{k}$. From the stability analysis it follows that the lowest quantum state $\mu_{1}$ is the only stable one. In some sense, this can be described by saying that in the spectral plane the normalized energy density flows towards lower wavenumbers. This completely agrees with the two cooperative effects of the inverse energy cascade established in the literature and the action of the dissipation. Note that the energy flow established here is solely a consequence of the presence of viscosity, since $\dot{Q}=0$ if $\nu=0$.

Now let us turn to the enstrophy density. In order to investigate its evolution, we need an expression for $\mathrm{d} \Lambda / \mathrm{d} t$. In a straightforward way it can be shown that

$$
\dot{\Lambda}(\omega)=-\frac{2 \nu}{\left(\int \omega^{2}\right)^{2}}\left[\int \omega^{2} \cdot \int(\Delta \omega)^{2}-\left(\int \nabla \omega\right)^{2}\right]+2 \frac{\int \Delta \omega v \cdot \nabla \omega}{\int \omega^{2}} .
$$

The expression $\int \Delta \omega \boldsymbol{v} \cdot \nabla \omega$ can be rewritten like $\int \nabla \omega \cdot\left(D_{x} v\right)_{\mathrm{s}} \nabla \omega$, where $\left(D_{x} v\right)_{\mathrm{s}}$ denotes the symmetric part of the Jacobian matrix of $v$ :

$$
\left(\mathrm{D}_{x} \boldsymbol{v}\right)_{\mathrm{s}}=\left(\begin{array}{ll}
\sigma_{11} & \tau_{12} \\
\tau_{21} & \sigma_{22}
\end{array}\right)
$$

with $\sigma_{11}=\left(v_{1}\right)_{x}, \sigma_{22}=\left(v_{2}\right)_{y}$ and $\tau_{12}=\tau_{21}=\frac{1}{2}\left[\left(v_{2}\right)_{x}+\left(v_{1}\right)_{y}\right]$.

Hence,

$$
\dot{\Lambda}(\omega)=\frac{-2 \nu}{\left(\int \omega^{2}\right)^{2}}\left[\int \omega^{2} \cdot \int(\Delta \omega)^{2}-\left(\int(\nabla \omega)^{2}\right)^{2}\right]+2 \frac{\int \nabla \omega \cdot\left(D_{x} v\right)_{s} \nabla \omega}{\int \omega^{2}} .
$$

Note that, unlike the change in $Q, \dot{A}$ depends on both the viscosity and the non-linearity of the problem.

Besides this, some other observations concerning (8.4) can be made. First of all, the expression in square brackets is non-negative for all $\omega$, and vanishes 
only if $\omega \in \mathrm{E}_{k}$ for some $k \in \mathbb{N}$. This can be shown in much the same way, the Cauchy-Schwartz inequality, as in lemma 6.1. Consequently, the first term on the right-hand side of (8.4) is non-positive and implies that the viscosity has the tendency to decrease the value of $A$, i.e. induces a flow in the spectral plane towards small wavenumbers. This first term is homogeneous of degree 0 , i.e. depends only on the spatial structure $\omega(t) /\|\omega(t)\|$. Unlike this, the second term on the right-hand side of (8.4) is homogeneous of degree 1 and so depends both on the magnitude as well as on the sign of $\omega$ : changing $v$ into $-\boldsymbol{v}$, the sign of this term changes (unlike that it vanishes, which is the case for Taylor's vortices for instance). Consequently, for appropriately chosen initial condition $\omega_{0}$, this term may be given either sign, in particular it may be positive, and have an arbitrarily large value. Hence, it may counterbalance the viscosity-driven motion towards lower wavenumbers. From this it may be concluded that $\dot{A}(\omega(t))$ can very well be positive for some interval of time. However, this cannot remain true for all $t$. In fact, since $\Lambda(\omega) \geqslant Q(\omega)$ for all $\omega$, and since $\Lambda(\omega(t))$ has the same limit as $Q(\omega(t))$ for $t \rightarrow \infty$ (see (6.5)), eventually $\Lambda(\omega(t))$ has decreased to a lowest value $\mu_{k}$ for some $k \in \mathbb{N}$.

Hence, for the spectral enstrophy density, the mean-squared wavenumber decreases to a lowest value ( $\mu_{1}$ for realistic flows) from above, but possibly in a non-monotonic way. In other terms, just as for the energy, there is a flow of the normalized enstrophy density towards smaller wavenumbers.

This decrease of the mean-squared wavenumber of the spectral enstrophy density is a result that may seem to be contradictory to the normal cascade of the enstrophy that is established in the literature. (See e.g. refs. 6, 7.) However, a plausible explanation for it must be that of the two counteracting effects that determine the value of enstrophy density at a certain wavenumber, viz. the normal cascade (i.e. the flow towards higher wavenumbers) and the discriminating dissipation (being larger at higher wavenumbers), the last one is dominating the first one. As far as we know, no such effect has ever been looked at, despite many numerical results available.

\section{Proof of the asymptotic results}

In this section we will prove propostion 7.1. The proof will be based on the following result which provides a measure for the distance of a function $\omega$ to a set $\mathrm{E}_{k}$ in terms of the difference $\Lambda(\omega)-Q(\omega)$. As before, $\mathrm{E}_{k}^{\perp}$ denotes the $\mathrm{L}_{2}$-orthogonal complement of $\mathrm{E}_{k}$.

Lemma 9.1. Let $\omega$ be decomposed as $\omega=\Omega+\xi$ with $\Omega \in \mathrm{E}_{k}$ and $\xi \in \mathrm{E}_{k}^{\prime}$. Then the following estimate holds: 


$$
A(\omega)-Q(\omega) \geqslant \sigma_{k} \rho\left(\frac{\|\xi\|}{\|\Omega\|}\right),
$$

where $\rho: \mathbb{R}_{+} \rightarrow \mathbb{R}_{+}$is the function $\rho(\varepsilon)=\varepsilon^{2} /\left(1+\varepsilon^{2}\right)^{2}$, and where $\sigma_{k}=$ $\left(\mu_{1} / \mu_{k}\right) \tau_{k}^{2}$ with $\tau_{k}^{2}$ a number that depends only on $\mu_{k}$ and its nearest neighbouring eigenvalue:

$$
\begin{aligned}
\tau_{k}^{2} & =\min \left[\frac{\left(\mu_{k}-\mu_{k-1}\right)^{2}}{\mu_{k-1}}, \frac{\left(\mu_{k+1}-\mu_{k}\right)^{2}}{\mu_{k+1}}\right] \text { for } k \geqslant 2, \\
\tau_{1}^{2} & =\frac{\left(\mu_{2}-\mu_{1}\right)^{2}}{\mu_{1}} .
\end{aligned}
$$

Proof. The orthogonal decomposition of $\omega$ implies that $E, W$ and $D$ are additive: $E(\omega)=E(\Omega)+E(\xi)$ and likewise for $W$ and $D$. Some elementary algebra then leads to

$$
\begin{aligned}
\Lambda(\omega)-Q(\omega)= & \frac{1}{E(\omega) W(\omega)}\left[\left\{D(\Omega) E(\Omega)-W^{2}(\Omega)\right\}\right. \\
& +\{E(\Omega) D(\xi)-2 W(\Omega) W(\xi)+D(\Omega) E(\xi)\} \\
& \left.+\left\{D(\xi) E(\xi)-W^{2}(\xi)\right\}\right]
\end{aligned}
$$

Since $D(\omega)=\mu_{k} W(\Omega)=\mu_{k}^{2} E(\Omega)$, the first expression in brackets vanishes, while the last expression is non-negative according to lemma 6.1. The middle term, which can be written like

$$
W(\Omega)\left\{\frac{1}{\mu_{k}} D(\xi)-2 W(\xi)+\mu_{k} E(\xi)\right\},
$$

can be analyzed by writing $\xi$ as in the foregoing section as a sum over the orthogonal functions $\Omega_{j}$ :

$$
\xi=\sum_{j}^{*} \alpha_{j} \Omega_{j}
$$

where now $\Sigma^{*}$ denotes summation overall $j$ for which $\lambda_{j} \neq \mu_{k}$ since $\xi \in \mathrm{E}_{k}^{\perp}$. Then the middle term reads

$$
W(\Omega) \cdot \frac{1}{2} \Sigma^{*}\left(\frac{\lambda_{j}}{\mu_{k}}-2+\frac{\mu_{k}}{\lambda_{j}}\right) \alpha_{j}^{2} .
$$

Since

$$
\frac{\lambda_{j}}{\mu_{k}}-2+\frac{\mu_{k}}{\lambda_{j}}=\frac{1}{\mu_{k} \lambda_{j}}\left(\lambda_{j}-\mu_{k}\right)^{2} \geqslant \frac{1}{\mu_{k}} \tau_{k}^{2}
$$


by definition of $\tau_{k}^{2}$, it follows by gathering together the pieces that

$$
A(\omega)-Q(\omega) \geqslant \frac{\tau_{k}^{2}}{\mu_{k}} \frac{W(\Omega)}{E(\omega) W(\omega)} W(\xi) .
$$

Finally, inserting the inequality $W(\omega) \geqslant \mu_{1} E(\omega)$ provides the required estimate:

$$
\Lambda(\omega)-Q(\omega) \geqslant \frac{\mu_{1}}{\mu_{k}} \tau_{k}^{2} \frac{W(\Omega) W(\xi)}{(W(\Omega)+W(\xi))^{2}}=\sigma_{k} \rho\left(\frac{\|\xi\|}{\|\Omega\|}\right) .
$$

Proof of proposition 7.1. In the proof of proposition 6.3 it was shown that for a solution $\omega \in I_{k}$ and a chosen time sequence $t_{n} \rightarrow \infty$ there exists $\hat{\omega} \in \mathrm{E}_{k}$ such that $\hat{\omega}\left(t_{n}\right) \rightarrow \hat{\omega}$ for some subsequence. Now we shall show that $\hat{\omega}(t) \rightarrow \hat{\omega}$ for $t \rightarrow \infty$, independent of the time sequence, thereby proving parts (i) and (ii) of 7.1 .

We write $\varepsilon(t)=\|\xi(t)\| /\|\Omega(t)\|$ and observe the following qualitative properties of the function $\rho$ entering in the lemma above: $\rho$ increases from $\rho=0$ for $\varepsilon=0$ to $\rho=\frac{1}{4}$ for $\varepsilon=1$, and then decreases to zero for $\varepsilon \rightarrow \infty$. Moreover, $\rho(\varepsilon) \geqslant \frac{1}{4} \varepsilon^{2}$ for all $\varepsilon \in(0,1)$.

Now, since $\Lambda(\omega(t))-Q(\omega(t)) \rightarrow 0$ as $t \rightarrow \infty$, it certainly holds that $A-Q \leqslant$ $\frac{1}{8} \sigma_{k}$ and hence $\rho(\varepsilon(t))<\frac{1}{8}$ for all $t$ sufficiently large, say $t>T$. Since, at the time sequence, $\varepsilon\left(t_{n}\right)<1$ for $n$ sufficiently large, the continuity of $t \rightarrow \varepsilon(t)$ and $\rho(\varepsilon(t))<\frac{1}{8}$ implies that $\varepsilon(t)<\varepsilon_{0}<1$, for all $t>T$, where $\varepsilon_{0}$ is such that $\rho\left(\varepsilon_{0}\right)=\frac{1}{8}$. Then the estimate $\frac{1}{4} \varepsilon^{2} \sigma_{k} \leqslant \sigma_{k} \rho(\varepsilon) \leqslant \Lambda(\omega)-Q(\omega) \rightarrow 0$ as $t \rightarrow \infty$ implies that $\varepsilon(t) \rightarrow 0$ for $t \rightarrow \infty$, which proves (i). The proof of part (ii) essentially reduces to show that there will be no motion in $\mathrm{E}_{k}$ (cf. remark 4.2). To that end let $V$ and $w$ denote the velocities corresponding to $\Omega$ and $\xi$, respectively. Then the equation for $\omega=\Omega+\xi$ can be written as

$$
\partial_{t} \Omega-V \cdot \nabla \Omega-\nu \Delta \Omega=-\partial_{t} \xi+w \cdot \nabla \Omega+V \cdot \nabla \xi+\nu \Delta \xi \text {. }
$$

Since $V \cdot \nabla \Omega=0$ and $-\Delta \Omega=\mu_{k} \Omega$, the first part (i) implies that $\Omega$ satisfies

$$
\partial_{r} \Omega+\nu \mu_{k} \Omega=o(\|\Omega\|),
$$

from which the required result follows.

To prepare for the estimate $\left(\mathrm{i}^{*}\right)$ we first derive an estimate for the approach of $Q$ to $\mu_{k}$. Using $E(\omega) \geqslant E(\Omega)=\left(1 / \mu_{k}\right) W(\Omega)$ it follows that

$$
Q(\omega)-\mu_{k}=\frac{1}{E(\omega)}\left[W(\omega)-\mu_{k} E(\omega)\right]=\frac{1}{E(\omega)}\left[W(\xi)-\mu_{k} E(\xi)\right]
$$




$$
\leqslant \frac{W(\xi)}{E(\Omega)}=\mu_{k} \varepsilon^{2}(t)
$$

Since the function $\rho$ satisfies for any $\alpha<1$ the estimate $\rho(\varepsilon) \geqslant \alpha \varepsilon^{2}$ for $\varepsilon$ sufficiently small, we derive from the fact that $\varepsilon(t) \rightarrow 0$ the estimate

$$
\Lambda(\omega)-Q(\omega) \geqslant \sigma_{k} \rho(\varepsilon) \geqslant \sigma_{k} \alpha \varepsilon^{2} \geqslant \alpha \frac{\sigma_{k}}{\mu_{k}}\left(Q-\mu_{k}\right),
$$

valid for any $\alpha<1$, provided $t$ is sufficiently large. Inserting this in the expression (6.1) for $\dot{Q}$ leads to

$$
\dot{Q} \leqslant-\alpha \nu \frac{\sigma_{k}}{\mu_{k}} Q\left(Q-\mu_{k}\right)
$$

and this gives the inequality

$$
Q \leqslant \mu_{k}\left[1-c_{1} \exp \left(-\alpha \nu \sigma_{k} t\right)\right]^{-1}
$$

for some constant $c_{1}>0$.

In the special case $k=1$, we have $W(\xi) \geqslant \mu_{2} E(\xi)$ and the relation (9.4) provides the estimate

$$
\left(1 \frac{\mu_{1}}{\mu_{2}}\right) W(\xi) \leqslant E(\omega)\left[Q(\omega)-\mu_{1}\right],
$$

and thus

$$
W(\xi) \leqslant c_{2} E(\omega) \exp (-\alpha \nu \sigma t)
$$

for some $c_{2}$. The asymptotic behaviour of $E(\omega)$ can be obtained with (5.2) from the fact that $Q-\mu_{1}$ is integrable over $\mathbb{R}_{+}$(as is seen from (9.5)):

$$
\dot{E}=-2 \nu W=-2 \nu Q E=-2 \nu \mu_{1} E-2 \nu\left(Q-\mu_{1}\right) E,
$$

i.e.

$$
E(\omega(t))=E(\omega(0)) \exp \left(-2 \nu \mu_{1} t\right) \exp \left[-2 \nu \int_{0}^{t}\left(Q-\mu_{1}\right) \mathrm{d} t\right]
$$

and thus

$$
E(\omega(0)) \exp \left(-2 \nu \mu_{1} t\right) \geqslant E(\omega) \geqslant c_{3} \exp \left(-2 \nu \mu_{1} t\right)
$$


for some $c_{3}>0$.

From (9.7) and (9.9) one first derives

$$
W(\Omega)=\mu_{1} E(\Omega) \geqslant c_{4} \exp \left(-2 \nu \mu_{1} t\right)
$$

and then

$$
\varepsilon^{2}(t)=\frac{W(\xi)}{W(\Omega)} \leqslant c_{5} \exp \left(-\alpha \nu \sigma_{1} t\right)
$$

which proves the estimate $\left(i^{*}\right)$ and completes the proof of proposition 7.1 .

\section{Acknowledgements}

The main ideas and results of this paper have been discussed at the Seminar on Mathematical Physics at the University of Twente in the spring 1986. I thank the participants of this seminar for their interest and some useful remarks.

\section{References}

1) F.P. Bretherton and D.B. Haidvogel, Two-dimensional turbulence above topography, J. Fluid Mech. 78 (1976) 129-154.

2) C. Foias and J.C. Saut, Linearization and normal form of the Navier-Stokes equations with potential forces, Ann. Inst. Henri Poincaré 4 (1987) 1-47.

3) C. Foias and J.C. Saut, Asymptotic behaviour, as $t \rightarrow \infty$ of solutions of Navier-Stokes equations and non-linear spectral manifolds, Indiana Univ. Math. J. 33 (1984) 459-477.

4) A. Hasegawa, Self-organization processes in continuous media, Adv. Phys. 34 (1985) 1-42.

5) Lord Kelvin, Mathematical and Physical Papers, vol. IV (Cambridge Univ. Press., Cambridge, 1910).

6) R.H. Kraichnan. Inertial ranges in two-dimensional turbulence, Phys. Fluids 10 (1967) 1417-1423.

7) R.H. Kraichnan and D. Montgomery, Two-dimensional turbulence, Rep. Prog. Phys. 43 (1980) 547-619.

8) P.D. Lax, Integrals of nonlinear equations of evolution and solitary waves, Comm. Pure Appl. Math. 21 (1968) 467-490.

9) J.C. McWillians, The emergence of isolated coherent vortices in turbulent flow, J. Fluid Mech. 146 (1984) 21-43.

10) P.B. Rhines, Waves and turbulence on a beta-plane, J. Fluid Mech. 69 (1975) 417-443.

11) G.T. Taylor, Phil. Mag. 46 (1923) 671.

12) Z. Yoshida. T. Uchida and N. Inoue, Self-organization of the reversed-field pinch quiescense, Phys. Fluids 27 (1984) 1785. 